\title{
POLYNOMIAL APPROXIMATION OF BERNSTEIN TYPE
}

\author{
BY \\ GILBERT STRANG(1)
}

1. Both positive and negative results are prominent in the theory of approximation to continuous functions by polynomials. The Bernstein polynomials, and for the periodic case the Fejér and de la Vallée-Poussin sums, are familiar instances of sequences of polynomial operators known to converge strongly to the identity. On the other hand, the usual Lagrange interpolation process cannot provide a convergent approximation for every function continuous on a given interval, however the points of interpolation are chosen.

Our present results are chiefly positive, and illustrate how convergent sequences of operators may still be constructed within the framework of Lagrange interpolation. Their starting-point is the interpretation of the Bernstein polynomials as the outcome of $n$-fold linear interpolation, as follows. Given a function $f$ in $C[0,1]$, first interpolate linearly over each subinterval $[m / n,(m+1) / n]$ to get values at the points $(m+x) / n, m=0,1, \cdots, n-1$. Using those values, interpolate over each interval $[(m+x) / n,(m+1+x) / n]$ for values at $(m+2 x) / n$, $m=0,1, \cdots, n-2$, and ultimately over the single interval

$$
[((n-1) x) / n,(1+(n-1) x) / n]
$$

for a value at $x$; this is easily seen to coincide with

$$
B_{n} f(x)=\sum_{r=0}^{n} f\left(\frac{r}{n}\right)\left(\begin{array}{l}
n \\
r
\end{array}\right) x^{r}(1-x)^{n-r} .
$$

The first aim of this paper is to establish that iterated interpolation of fixed order $2 N-1$ succeeds for every $N$. It is convenient to describe this type of interpolation in the following terms. Let $Q_{N}(x, \theta)$ be the Lagrange interpolating polynomial of degree $2 N-1$ in $x$ which agrees with $e^{i \theta x}$ at the points $x=1-N$, $2-N, \cdots, N$, and let

$$
\left(Q_{N}\right)^{n}=\sum_{=n(1-N)}^{n N} c_{r n}^{(N)}(x) e^{i r \theta} .
$$

Then for a function $f$ in $C[1-N, N]$ we define the operator

$$
B_{n}^{(N)} f(x)=\sum_{r=n(1-N)}^{n N} c_{r n}^{(N)}(x) f\left(\frac{r}{n}\right)
$$

Received by the editors October 27, 1961.

(1) The preparation of this paper was supported by a N.A.T.O. Fellowship and by NSF Contract G 19864. 
Of course

$$
Q_{1}=1-x+x e^{i \theta}, c_{r n}^{(1)}=\left(\begin{array}{l}
n \\
r
\end{array}\right) x^{r}(1-x)^{n-r}
$$

and $B_{n}^{(1)} f=B_{n} f$.

We shall state our principal result as Theorem 1, although the final steps in its proof are not to be found until §3. That proof hinges on a question of quite general interest in analysis, which is formulated in $\S 4$; we point out some of the special cases which have been solved, and have to leave it at that. The last section is devoted to a closer study of the degree of approximation and asymptotic form of the operators $B_{n}^{(N)}$. Our theorems reproduce in the case $N=1$ much of the theory of Bernstein polynomials in the real domain created by Bernstein, Popoviciu, Voronowskaja, Lorentz, and others (see [5]). The proofs are necessarily quite different, since the coefficients $c_{r n}$ are no longer all positive once $N>1$. Our new approach contributes an exact value for the constant in one of the standard inequalities of the Bernstein theory.

Theorem 1 can be translated into a statement about finite difference approximations to hyperbolic partial differential equations with constant coefficients. It says that certain of the most accurate explicit methods using an even number of points are stable in the maximum norm (cf. [8], where $L_{2}$ stability is proved). We hope to give elsewhere a full discussion from this point of view. The corresponding problem for an odd number of points, that is, for interpolation of even order, is still to be decided.

2. THEOREM 1. For every fixed $N$, and every function $f$ in $C[1-N, N]$, $\lim _{n \rightarrow \infty} B_{n}^{(N)} f=f$ uniformly on the subinterval $0 \leqq x \leqq 1$.

There are two facts to be established in the proof:

(i) $B_{n}^{(N)} f$ converges uniformly to $f$ on $[0,1]$ for some dense subset of $C[1-N, N]$.

(ii) $\left\|B_{n}^{(N)}\right\|=\sup _{0 \leqq x \leqq 1} \sum_{n(1-N)}^{n N}\left|c_{r n}^{(N)}(x)\right|$ has, for each fixed $N$, a bound independent of $n$.

We begin with several lemmas which contain the proof of (ii). The argument is entirely straightforward, but this is partly concealed by the fact that we also obtain in these lemmas the more general inequalities needed in $\S 5$.

Everything depends on some fundamental properties of the "generating polynomials" $Q_{N}$. Polynomials of this type were introduced in our earlier paper [8] and we look there for the first lemma.

LEMMA 1. If $\theta$ is real and $0 \leqq x \leqq 1$, then $\left|Q_{N}\right| \leqq 1$; the strict inequality $\left|Q_{N}\right|<1$ holds in case $0<x<1$ and $\theta \neq 0(\bmod 2 \pi)$.

Proof. This is the one point where our choice of $[0,1]$ as the basic interval is inconvenient. It is easy to shift to an interval symmetric about the origin by 
defining $q_{N}(y, \theta)$ to be the polynomial of degree $2 N-1$ in $y$ which satisfies $q_{N}=e^{i \theta y}$ for $y=1-2 N, 3-2 N, \cdots, 2 N-1$. Then clearly

$$
Q_{N}(x, \theta)=q_{N}(2 x-1, \theta / 2) e^{i \theta / 2}
$$

since they agree at $x=1-N, 2-N, \cdots, N$. We must now refer the reader to the proof of Theorem 1 in [8], where we show that $\left|q_{N}(y, \theta)\right| \leqq 1$ for $-1 \leqq y \leqq 1$, and $\left|q_{N}\right|<1$ when $-1<y<1$ and $\theta \neq 0(\bmod \pi)$. The lemma then follows immediately from (3).

The second lemma provides a more precise result near $\theta=0$, and is the key step in showing that the convergence is uniform in $x$.

LEMMA 2. There are positive constants $A, A_{0}, A_{1}, \ldots$, depending only on $N$ such that, for $0 \leqq x \leqq 1$ and $-\pi \leqq \theta \leqq \pi$,

$$
\begin{aligned}
\left|e^{i \theta x}-Q_{N}\right| & \leqq A_{0} x(1-x) \theta^{2 N}, \\
\left|\frac{d^{k}}{d \theta^{k}}\left(Q_{N} e^{-i \theta x}\right)\right| & \leqq A_{k} x(1-x) \theta^{2 N-k^{\prime}}, \quad k=1,2, \cdots, \\
\left|Q_{N}\right| & \leqq \exp \left(-A x(1-x) \theta^{2 N}\right)
\end{aligned}
$$

where $k^{\prime}=\min (k, 2 N)$.

Proof. Look first at the difference $e^{i \theta x}-Q_{N}(x, \theta)$, expanded in powers of $\theta$. The coefficient of $\theta^{k}, k<2 N$, must vanish identically, being a polynomial of degree $2 N-1$ in $x$ with zeros at $x=1-N, \cdots, N$ (where $Q_{N}=e^{i \theta x}$ by definition). The coefficient of $\theta^{2 N}$ has degree $2 N$, with leading coefficient $(-1)^{N} /(2 N)$ !, and is therefore given by

$$
\rho_{N}(x)=\frac{(-1)^{N}}{(2 N) !} \prod_{1-N}^{N}(x-j) .
$$

Thus $e^{i \theta x}-Q_{N}$, or equally well $Q_{N} e^{-i \theta x}-1$, is an entire function of $x$ and $\theta$, with zeros at $x=0$ and $x=1$ for all $\theta$, and a $2 N$-fold zero at $\theta=0$ for all $x$. This is enough knowledge to settle (4) and (5), but (6) is a little more delicate. We want to establish that

$$
h(x, \theta)=\frac{1-\left|Q_{N}\right|}{x(1-x) \theta^{2 N}}
$$

is strictly positive in the rectangle $0 \leqq x \leqq 1,-\pi \leqq \theta \leqq \pi$. By the first lemma, $h>0$ except perhaps when $x=0, x=1$, or $\theta=0$. The possibility $\theta=0$ is eliminated by looking at $h(x, 0)=\rho_{N} / x(1-x)$, which is certainly positive when $0 \leqq x \leqq 1$. To handle the lines $x=0$ and $x=1$, recall that $Q_{N}(0, \theta)=1$, $Q_{N}(1, \theta)=e^{i \theta}$, and $Q_{N}=\sum_{1-N}^{N} c_{r 1}(x) e^{i r \theta}$. By direct calculation 


$$
\frac{\partial\left|Q_{N}\right|}{\partial x}(0, \theta)=\sum_{1-N}^{N} c_{r 1}^{\prime}(0) \cos r \theta, \frac{\partial\left|Q_{N}\right|}{\partial x}(1, \theta)=\sum_{1-N}^{N} c_{r 1}^{\prime}(1) \cos (r-1) \theta .
$$

Both of these are trigonometric polynomials of order $N$, with zeros of order $2 N$ at $\theta=0$, and so neither can vanish elsewhere in $-\pi \leqq \theta \leqq \pi$. Therefore $1-\left|Q_{N}\right|$ has only simple zeros at $x=0$ and $x=1$, away from $\theta=0$. By continuity, $h$ is positive throughout the rectangle and attains its lower bound, which we take as $A$. Then $\left|Q_{N}\right| \leqq 1-A x(1-x) \theta^{2 N}$, and (6) follows from the inequality $1-u$ $\leqq \exp (-u)$.

3. We come now to estimate the coefficients $c_{r n}^{(N)}=c_{r n}^{(N)}(x)$ which appear in the expansion $\left(Q_{N}\right)^{n}=\sum c_{r n}^{(N)} e^{i r \theta}$. It should be said that only the cases $k=0$ and $k=2$ below, for which $k=k^{\prime}=k^{\prime \prime}$, will be wanted in the proof of Theorem 1 . It is Theorem 3 which demands that we extend the lemma to higher values of $k$, and so become involved in the special treatment required when $k>2 N$.

We introduce the definition $z=[n x(1-x)]^{1 / 2 N}$.

LEMMA 3. There are constants $B_{0}, B_{1}, \cdots$, depending only on $N$, such that for $0 \leqq x \leqq 1$ and $n x \neq r$,

$$
\left|c_{r n}^{(N)}\right| \leqq \frac{B_{k} z^{k^{\prime \prime}-1}}{|n x-r|^{k}}, \quad k=0,1, \cdots
$$

where $k^{\prime \prime}=k$ when $z \geqq 1, k^{\prime \prime}=k^{\prime}$ when $z<1$. There is also the universal bound $\left|c_{r n}^{(N)}\right| \leqq 1$ when $0 \leqq x \leqq 1$.

Proof. Suppose that $0<x<1$, the excluded cases being trivial, and consider the formula

$$
c_{r n}^{(N)}=\frac{1}{2 \pi} \int_{-\pi}^{\pi}\left(Q_{N}\right)^{n} e^{-i r \theta} d \theta=\frac{1}{2 \pi} \int_{-\pi}^{\pi}\left(Q_{N} e^{-i \theta x}\right)^{n} e^{i \theta(n x-r)} d \theta .
$$

Using the fact that $\left|Q_{N}\right| \leqq 1$, the estimate $\left|c_{r n}^{(N)}\right| \leqq(1 / 2 \pi) \int_{-\pi}^{\pi} d \theta=1$ is trivial. If we apply instead the inequality (6) and change variables to $\phi=z \theta$, the result is

$$
\left|c_{r n}^{(N)}\right| \leqq \frac{1}{2 \pi} \int_{-\pi}^{\pi} \exp \left(-n A x(1-x) \theta^{2 N}\right)<\frac{1}{2 \pi z} \int_{-\infty}^{\infty} \exp \left(-A \phi^{2 N}\right) d \phi=\frac{B_{0}}{z}
$$

The estimates for $k>0$ require $k$ integrations by parts of the second integral in (8). Although the parts don't have period $2 \pi$, their product always does, and the integrated terms vanish to give

$$
\begin{aligned}
c_{r n}^{(N)} & =\frac{1}{2 \pi} \int_{-\pi}^{\pi} \frac{d^{k}}{d \theta^{k}}\left(Q_{N} e^{-i \theta x}\right)^{n} \frac{e^{i \theta(n x-r)}}{i k(n x-r)^{k}} d \theta \\
\left|c_{r n}^{(N)}\right| & \leqq \frac{1}{2 \pi|n x-r|^{k}} \int_{-\pi}^{\pi}\left|\frac{d^{k}}{d \theta^{k}}\left(Q_{N} e^{-i \theta x}\right)^{n}\right| d \theta
\end{aligned}
$$


One is forced to expand the derivative and estimate the terms separately, a typical term being an integer multiple of

$$
n(n-1) \cdots(n-t+1) \frac{d^{k_{1}}}{d \theta^{k_{1}}}\left(Q_{N} e^{-i \theta x}\right) \cdots \frac{d^{k_{t}}}{d \theta^{k_{t}}}\left(Q_{N} e^{-i \theta x}\right) \cdot\left(Q_{N} e^{-i \theta x}\right)^{n-t}
$$

where $\Sigma_{1}^{t} k_{i}=k$ and so $1 \leqq t \leqq k$. (The $k_{i}$ need not be distinct.) Using the notation of the previous lemma, we define $K=\Sigma_{1}^{t} k_{i}^{\prime}$, so that $K \leqq 2 N t$ and $k^{\prime} \leqq K \leqq k$. Now by the estimates of Lemma 2 our typical term is bounded by

$$
M=\text { constant } \cdot(z \theta)^{2 N t} \theta^{-K} \exp \left(-n A x(1-x) \theta^{2 N}\right)
$$

with the constant depending only on $N$ and $k$. With the same change of variable $\phi=z \theta$, we have

$$
\begin{aligned}
\int_{-\pi}^{\pi} M d \theta & <\text { constant } \cdot z^{K-1} \int_{-\infty}^{\infty} \phi^{2 N t-K} \exp \left(-A \phi^{2 N}\right) d \phi \\
& <\text { constant } \cdot z^{K-1} \leqq \text { constant } \cdot z^{k \prime-1} .
\end{aligned}
$$

Thus each of finitely many terms in the expansion of the derivative yields the right bound; combining them, the third lemma is established.

LEMMA 4. There are constants $C_{0}, C_{1}, \cdots$, depending only on $N$, such that for $0 \leqq x \leqq 1$

$$
\sum_{r=n(1-N)}^{n N}|n x-r|^{m}\left|c_{r n}^{(N)}\right| \leqq C_{m} z^{(m+2)^{\prime \prime-2}} \leqq C_{m} n^{m / 2 N} .
$$

Proof. The second inequality is immediate, since $n x(1-x)<n$ and $(m+2)^{\prime \prime}-2 \leqq m$, and puts the lemma into its most useful form. To demonstrate the first inequality, split the sum on the left into two parts, according to whether $r$ satisfies $|n x-r| \leqq z$ or not.

In the first part, where $|n x-r| \leqq z$, suppose first that $z<1$. Then this part contains no more than two terms, and (using $\left.\left|c_{r n}^{(N)}\right| \leqq 1\right)$ contributes no more than $2 z^{m}$. If $z \geqq 1$, the contribution of the first part is less than

$$
\frac{B_{0}}{z} \sum_{|n x-r| \leqq z}|n x-r|^{m}<\text { constant } \cdot z^{m} \text {. }
$$

The second part of the sum adds less than

$$
\sum_{|n x-r|>z} \frac{B_{m+2} z^{(m+2)^{n-1}}}{|n x-r|^{2}}<\text { constant } \cdot z^{(m+2) n-2} .
$$

The uniformity appears suspect for small $z$, but in fact $z<|n x-r|<1 / 2$ never occurs, since $\min (n x, n(1-x)) \leqq 2 n x(1-x)<z$ whenever $z<1 / 2$.

This establishes the fourth lemma, and leaves very little to be done in completing the proof of the principal theorem.

Proof of Theorem 1. We use inequality (4) to verify that for any real $\alpha$, $\lim _{n \rightarrow \infty} B_{n}^{(N)} e^{i \alpha x}=e^{i \alpha x}$ uniformly for $0 \leqq x \leqq 1$ : 


$$
B_{n}^{(N)} e^{i \alpha x}=\sum c_{r n}^{(N)} e^{i \alpha r / n}=\left[Q_{N}(x, \alpha / n)\right]^{n}=\left[e^{i \alpha x / n}+O\left(\left(\frac{\alpha}{n}\right)^{2 N}\right)\right]^{n} \rightarrow e^{i \alpha x}
$$

Theorem 1 then holds for any finite linear combination $g$ of exponentials $e^{i \alpha x}$ (or of $\sin \alpha x$ and $\cos \alpha x$, if we follow the convention of approximation theory in taking the elements of $C$ to be real-valued functions).

Given any $f$ in $C[1-N, N]$, and any $\varepsilon>0$, there is a linear combination $g$ such that $|f-g|<\varepsilon$ for $1-N \leqq x \leqq N$, so that by Lemma 4

$$
\left|B_{n}^{(N)} f-f\right| \leqq\left|B_{n}^{(N)} g-g\right|+\left(C_{0}+1\right) \varepsilon<\left(C_{0}+2\right) \varepsilon
$$

for all sufficiently large $n$, uniformly for $0 \leqq x \leqq 1$.

4. With the proof of Theorem 1 now complete, we pause for a moment to consider the problem whose solution for the particular polynomials $Q_{N}$ gave us that theorem. We state the discrete form, although the corresponding problem for $L_{1}$ is equally important. The algebra of absolutely convergent trigonometric series $f(\theta)=\sum_{-\infty}^{\infty} a_{j} e^{i j \theta}$ is normed by $\|f\|=\Sigma\left|a_{j}\right|$. For what elements $f$ is the sequence $\left\|f^{n}\right\|$ bounded?

The set of such elements is clearly convex, but it is neither open nor closed. By a theorem of Beurling $\left[6\right.$, p. 428] $\lim \left\|f^{n}\right\|^{1 / n}=\sup |f(\theta)|$; this leaves in doubt only the case when $\sup |f(\theta)|=1$. Using very elegant, and yet wholly elementary, arguments, Beurling and Helson have answered the question when $|f(\theta)|=1$ for all $\theta$. Among such functions in $\mathscr{A}$ only the exponentials $f=\varepsilon e^{i j \theta},|\varepsilon|=1$, have the property that $\left\|f^{n}\right\|=O$ (1). For the rest, the order of growth is to be determined, and we have thought it worthwhile to carry through in this section its determination for one of the most interesting examples.

In dealing with the $Q_{N}$, on the other hand, we could not avoid finding a large, but very ramshackle, class of series that do have bounded powers. Loosely speaking, $\left\|f^{n}\right\|$ is bounded when a smooth function $f$ in $\mathscr{A}$ has an odd degree of osculation with the unit circle. More precisely, we consider the function

$$
g\left(\theta, \theta_{k}\right)=\frac{f(\theta)}{f\left(\theta_{k}\right)} \exp \left[\frac{-f^{\prime}\left(\theta_{k}\right)}{f\left(\theta_{k}\right)}\left(\theta-\theta_{k}\right)\right]
$$

THEOREM 2. If $f$ is twice continuously differentiable, $|f|<1$ for $-\pi \leqq \theta \leqq \pi$ except at a finite set of points $\theta_{1}, \cdots, \theta_{t}$, and if there exist positive integers $N_{k}$ and positive constants $M, M^{\prime}$, $\delta$, such that if $\left|\theta-\theta_{k}\right|<\delta$, then $\left|g\left(\theta, \theta_{k}\right)\right|$ $\leqq 1-M\left(\theta-\theta_{k}\right)^{2 N_{k}}$ and $\left|g^{\prime \prime}\left(\theta, \theta_{k}\right)\right| \leqq M^{\prime}\left(\theta-\theta_{k}\right)^{2 N_{k}-2}, 1 \leqq k \leqq t$, then $\left\|f^{n}\right\|$ is bounded. However, there exists an element of $\mathscr{A}$ such that $|f| \leqq 1$ but $\lim \sup \left\|f^{n}\right\|$ $=$ constant $n^{1 / 2}$.

Proof. To establish the first statement, choose a real $C^{\infty}$ function $h(\theta)$ in $\mathscr{A}$ such that $h \equiv 1$ for $|\theta| \leqq \Delta, h \equiv 0$ for $2 \Delta \leqq|\theta| \leqq \pi$. Define $f_{k}(\theta)=h\left(\theta-\theta_{k}\right) f(\theta)$ and $F(\theta)=f(\theta)-\sum f_{k}(\theta)$. If $\Delta$ is small enough, then $f_{k} f_{k^{\prime}} \equiv 0$ whenever $k \neq k^{\prime}$; this means that $\sup |F|<1$, so that $\left\|F^{n}\right\|$ is bounded by Beurling's 
theorem. For each $k$, the proof that $\left\|\left(F+f_{k}\right)^{n}\right\|$ is bounded is exactly that given above for the $Q_{N}$. Finally, $f^{n}=\left(F+\sum f_{k}\right)^{n}=\sum\left(F+f_{k}\right)^{n}-(t-1) F^{n}$, so by the triangle inequality $\left\|f^{n}\right\|$ is bounded.

The example we need for the second part of the theorem is provided by $f(\theta)=\exp (i \beta \sin \theta)$. Since the Bessel coefficients satisfy

$$
J_{p}(\beta n)=\frac{1}{2 \pi} \int_{-\pi}^{\pi} e^{i \beta n \sin \theta} e^{-i p \theta} d \theta
$$

we may use the uniform asymptotic expansions derived by Langer [4] and displayed in [2, p. 89] to show that $\left\|f^{n}\right\|=\Sigma_{p}\left|J_{p}(\beta n)\right|$ is unbounded for some $\beta$. Suppose we consider only $n$ even, $1 \leqq p \leqq n / 2$, and $1 \leqq \beta \leqq 2$. Defining $w=\left(\left(\beta^{2} n^{2} / p^{2}\right)-1\right)^{1 / 2}$, and expanding asymptotically the Bessel functions of order $1 / 3$ which appear in Langer's formula, we get

$$
\begin{aligned}
& J_{p}(\beta n)=\left(\frac{2}{\pi p w}\right)^{1 / 2} \cos \left(p\left(w-\tan ^{-1} w\right)-\pi / 4\right)+O\left(n^{-3 / 2}\right)+O\left(p^{-4 / 3}\right) . \\
& \sum_{p=1}^{n / 2}\left|J_{p}(\beta n)\right|>(\pi n)^{-1 / 2} \sum_{p=1}^{n / 2}\left|\cos \left(p\left(w-\tan ^{-1} w\right)-\pi / 4\right)\right|+\text { constant. }
\end{aligned}
$$

Denoting by $S_{n}(\beta)$ the sum of cosines on the right, we have now to show that for some constants $\beta$ and $\mu, S_{n}>\mu n$ for infinitely many $n$. There is no reason to think otherwise, but the argument is not interesting and we give it only in outline: one finds a $\mu$ for which always $\int_{1}^{2}\left|\cos \left(p\left(w-\tan ^{-1} w\right)-\pi / 4\right)\right| d \beta>4 \mu$, so that $\int_{1}^{2} S_{n} d \beta>2 \mu n$. Since $S_{n}<n$, the set of those $\beta$ for which $S_{n}>\mu n$ must have, for each $n$, measure exceeding $\mu(1-\mu)^{-1}$. Some $\beta$ therefore belongs to infinitely many of these sets, and we have shown that limsup $\left\|f^{n}\right\| \geqq \operatorname{constant} \sqrt{n}$.

The reverse estimate is easy; first of all

$$
\sum_{p=-8 n}^{8 n-1}\left|J_{p}(\beta n)\right| \leqq 4 \sqrt{n} \quad \sum J_{p}^{2}(\beta n) \leqq \frac{2 \sqrt{n}}{\pi} \int|f(\theta)|^{2} d \theta=4 \sqrt{n} .
$$

The remaining terms in $\Sigma\left|J_{p}(\beta n)\right|$, for which $|p| \geqq 8 n$, contribute less than some absolute constant; this follows from the estimate $[2$, p. 14]

$$
\left|J_{p}(x)\right| \leqq \frac{1}{p !}\left(\frac{x}{2}\right)^{p}
$$

5. We begin a more detailed study of the polynomial operators $B_{n}^{(N)}$ by computing their degree of approximation. As one would expect, the Bernstein operators $B_{n}^{(1)}$ are the least accurate in the sequence. We denote by $\omega_{0}$ the modulus of continuity of a given function $f$ in $C[1-N, N]$; in case the derivative $f^{(q)}$ exists and is continuous, its modulus of contuinity shall be $\omega_{q}$.

Theorem 3. There are constants $D_{0}, D_{1}, \cdots, D_{2 N-1}$, depending only on $N$, such that if $f^{(q)}$ is in $C[1-N, N]$ and $0 \leqq x \leqq 1$, then 


$$
\left|B_{n}^{(N)} f(x)-f(x)\right| \leqq D_{q} n^{q((1 / 2 N)-1)} \omega_{q}\left(n^{((1 / 2 N)-1)}\right), \quad 0 \leqq q \leqq 2 N-1 .
$$

Proof. The mean-value theorem can be written in the form

$$
f\left(x^{\prime}\right)-f(x)=\sum_{p=1}^{q}{\frac{\left(x^{\prime}-x\right)^{p}}{p !}}^{(p)}(x)+{\frac{\left(x^{\prime}-x\right)^{q}}{q !}}^{q}\left[f^{(q)}(\xi)-f^{(q)}(x)\right]
$$

where $\xi$ lies between $x$ and $x^{\prime}$, the sum disappearing if $q=0$. For any $\delta>0$,

$$
\left|f^{(q)}(\xi)-f^{(q)}(x)\right| \leqq \omega_{q}(|\xi-x|) \leqq \omega_{q}\left(\left|x^{\prime}-x\right|\right) \leqq\left(\left|x^{\prime}-x\right| \delta^{-1}+1\right) \omega_{q}(\delta) .
$$

We also need the fact that $B_{n}^{(N)} h \equiv h$ for all $n$, whenever $h$ is a polynomial of degree less than $2 N$. This follows directly from the original description of $B_{n}^{(N)}$ as a composition of interpolations of order $2 N-1$, each of which will be exact for $h$. More formally, we compare the coefficients of $\alpha^{k}, k<2 N$, in the relation (13)

$$
B_{n}^{(N)} e^{i \alpha x}=\left[e^{i \alpha x / n}+O\left(\left(\frac{\alpha}{n}\right)^{2 N}\right)\right]^{n} .
$$

This gives $B_{n}^{(N)}\left(i x^{k} / k !\right) \equiv\left(i x^{k} / k !\right)$, and so $B_{n}^{(N)} h \equiv h$ by linearity. Now

$$
\begin{aligned}
\left|B_{n}^{(N)} f(x)-f(x)\right| & =\left|\sum_{r}\left(f\left(\frac{r}{n}\right)-f(x)\right) c_{r n}^{(N)}(x)\right| \\
\leqq & \sum_{p=1}^{q}\left|\sum_{r}\left(\frac{r}{n}-x\right)^{p} \frac{f^{(p)}(x)}{p !} c_{r n}^{(N)}\right| \\
& +\sum_{r}\left|\frac{r}{n}-x\right|^{q}\left(\left|\frac{r}{n}-x\right| \delta^{-1}+1\right) \frac{\omega_{q}(\delta)}{q !}\left|c_{r n}^{(N)}\right| \\
\leqq & \frac{\omega_{q}(\delta)}{q !}\left(C_{q+1} n^{(q+1)((1 / 2 N)-1)} \delta^{-1}+C_{q} n^{q((1 / 2 N)-1)}\right) \\
& =\left(\frac{C_{q+1}+C_{q}}{q !}\right) n^{q((1 / 2 N)-1)} \omega_{q}\left(n^{(1 / 2 N)-1}\right)
\end{aligned}
$$

using Lemma 4 for the last inequality and $\delta=n^{(1 / 2 N)-1}$. The theorem therefore holds with $D_{q}=\left(C_{q+1}+C_{q}\right)(q !)^{-1}$.

The important cases of Theorem 3 are the extreme ones. At the lower end, the conclusion

$$
\left|B_{n}^{(N)} f-f\right| \leqq D_{0} \omega_{0}\left(n^{(1 / 2 N)-1}\right)
$$

should be compared with Jackson's theorem that there are polynomials $P_{n}^{(N)}$, of equal degree $(2 N-1) n$ with $B_{n}^{(N)} f$, for which $\left|P_{n}^{(N)}-f\right|=O\left(\omega_{0}\left(n^{-1}\right)\right)$.

The result at the other extreme is that when $f^{(2 N)} N^{-1}$ is Lipschitz continuous with exponent 1 , i. e., $\omega_{2 N-1}(\delta) \leqq M \delta$, then

$$
\left|B_{n}^{(N)} f-f\right| \leqq D_{2 N-1} M n^{1-2 N} .
$$

For the Bernstein case $N=1$, this was recently shown by deLeeuw [3] to be impossible to improve in the sense that $\left|B_{n} f-f\right|=o\left(n^{-1}\right)$ can hold only for 
linear $f$. We have not undertaken to prove the corresponding conjecture when $N>1$. However, it is remarkable that (24) can be demonstrated using only the bound $\left\|B_{n}{ }^{(N)}\right\| \leqq C_{0}=C_{0}{ }^{(N)}$, without the cases $m>0$ of Lemma 4 which the weaker conclusions of Theorem 3 seem to require. The proof leads naturally to $1 / 8$ as the best possible value for $D_{1}$ in the classical case $N=1$, which seems to be a novelty; a briefer proof of this result is easily given.

THEOREM 4. If $f^{(2 N-1)}$ is in $C[1-N, N]$ and $\omega_{2 N-1}(\delta) \leqq M \delta$, then for $0 \leqq x \leqq 1$,

$$
\begin{aligned}
\left|B_{n}^{(1)} f(x)-f(x)\right| & \leqq \frac{M}{8 n}, \\
\left|B_{n}^{(N)} f(x)-f(x)\right| & \leqq C_{0}^{(N)} M n^{1-2 N}, \quad N>1 .
\end{aligned}
$$

Equality holds in (25) (at $x=1 / 2)$ if and only if $f(x)= \pm(M / 2) x^{2}+\alpha x+\beta$, $\alpha, \beta, n$ arbitrary.

Proof. If $f(x)= \pm(M / 2) x^{2}+\alpha x+\beta$, one quickly calculates

$$
\left|B_{n}^{(1)} f-f\right|=\frac{M x(1-x)}{2 n}=\frac{M}{8 n} \text { at } x=\frac{1}{2} .
$$

For the converse, we first inquire into the maximum error possible in linear interpolation, using abscissas $x_{1}$ and $x_{2}$, given that $x_{1} \leqq x \leqq x_{2}, x_{2}-x_{1}=n^{-1}$, and $\omega_{1}(\delta) \leqq M \delta$. It is an easy exercise to show that

$$
\left|\frac{x_{2}-x}{x_{2}-x_{1}} f\left(x_{1}\right)+\frac{x-x_{1}}{x_{2}-x_{1}} f\left(x_{2}\right)-f(x)\right| \leqq \frac{M}{8 n^{2}}
$$

with equality possible only for quadratic $f$. More generally, the error in interpolation of order $2 N-1$, using the abscissas $x_{1}, \cdots, x_{2 N}$, separated by $n^{-1}$, is given in divided difference notation by

$$
\begin{aligned}
\mid f & {\left[x, x_{1}, \cdots, x_{2 N}\right] \prod\left(x-x_{i}\right) \mid } \\
& =\left[\frac{f\left[x, x_{1}, \cdots, x_{2 N-1}\right]}{x_{1}-x_{2 N}}-\frac{f\left[x, x_{2}, \cdots, x_{2 N}\right]}{x_{1}-x_{2 N}}\right] \prod\left|x-x_{i}\right| \\
& =\left|\frac{f^{(2 N-1)}\left(\xi_{1}\right)-f^{(2 N-1)}\left(\xi_{2}\right)}{x_{1}-x_{2 N}}\right| \frac{\prod\left|x-x_{i}\right|}{(2 N-1) !} \leqq M n^{-2 N}
\end{aligned}
$$

supposing that $x_{1} \leqq x \leqq x_{2 N}$ and $\omega_{2 N-1}(\delta) \leqq M \delta$. (No trouble has been taken to get the best bound.) Therefore the first stage of the interpolations which make up $B_{n}^{(N)} f(x)$, namely those using the abscissas $(m+1-N) / n,(m+2-N) / n$, $\cdots,(m+N) / n$ to produce values at $(m+x) / n, m=(n-1)(1-N),(n-1)$ $(1-N)+1, \cdots,(n-1) N$, introduces errors of magnitude not more than $M / 8 n^{2}$, when $N=1$, or $M n^{-2 N}$, when $N>1$. These contribute to the final error in $B_{n}^{(N)} f(x)$ with an amplification factor not exceeding $C_{0}^{(N)}$. In particular, since 
$C_{0}^{(1)}=1$, the first-stage errors can contribute $M / 8 n^{2}$ to the net error only when $f$ is piecewise quadratic, and when the individual errors are all of the same sign, implying that $f$ is a genuine quadratic. Arguing in this way at each of the $n$ stages of interpolation, we find the global error bounds (25) and (26).

The next theorem is a generalization of an asymptotic formula due in the case $N=1$ to Voronowskaja. Its proof can be modelled faithfully on that given by Lorentz, so we omit the details.

THEOREM 5. Suppose that for a function $f$ in $C[1-N, N]$, the derivative $f^{(2 N)}$ exists at some point $x, 0 \leqq x \leqq 1$. Let $g(x)=x^{2 N}$. Then as $n \rightarrow \infty$

$$
B_{n}^{(N)} f(x)-f(x) \cong \frac{f^{(2 N)}(x)}{(2 N) !}\left(B_{n}^{(N)} g(x)-g(x)\right)=\frac{-f^{(2 N)}(x) \prod_{1-N}^{N}(x-j)}{n^{2 N-1}(2 N) !}
$$

Schoenberg has illustrated in [7] how to find an asymptotic result in another direction. His idea, employed in the study of smoothing operators, can be applied here to give the asymptotic form of the coefficients $c_{r n}^{(N)}$ which define $B_{n}^{(N)}$. When $N=1$, and the $c_{r n}$ are binomial coefficients, this form is familiar from the Laplace-de Moivre Theorem of probability theory.

THEOREM 6. For each $N$ and each $x$ in the open interval $0<x<1$, let

$$
G_{N}(s)=\frac{1}{2 \pi} \int_{-\infty}^{\infty} e^{-t^{2 N}-i s t} d t, \quad \rho_{N}(x)=\frac{(-1)^{N}}{(2 N) !} \prod_{1-N}^{N}(x-j) .
$$

Suppose $\varepsilon>0$. Then uniformly in $r$

$$
c_{r n}^{(N)}(x)=\left(n \rho_{N}\right)^{-1 / 2 N} G_{N}\left(\left(n \rho_{N}\right)^{-1 / 2 N}(r-n x)\right)+O\left(n^{-1 / N+\varepsilon}\right) .
$$

Proof. The two quantities to be compared are

$$
\begin{gathered}
c_{r n}^{(N)}=\frac{1}{2 \pi} \int_{-\pi}^{\pi}\left(Q_{N} e^{-i \theta x}\right)^{n} e^{-i \theta(r-n x)} d \theta \\
\left(n \rho_{N}\right)^{-1 / 2 N} G_{N}\left(\left(n \rho_{N}\right)^{-1 / 2 N}(r-n x)\right) \\
=\frac{1}{2 \pi} \int_{-\infty}^{\infty} \exp \left[-n \rho N \theta^{2 N}\right] \exp [-i \theta(r-n x)] d \theta .
\end{gathered}
$$

Recalling from Lemma 2 that $Q_{N} e^{-i \theta x}=1-\rho_{N} \theta^{2 N}+O\left(\theta^{2 N+1}\right)$, and using the inequality $\left|a^{n}-b^{n}\right| \leqq n|a-b|$, valid when $|a| \leqq 1,|b| \leqq 1$, we find

$$
\begin{aligned}
\left|\left(Q_{N} \exp [-i \theta x]\right)^{n}-\exp \left[-n \rho_{N} \theta^{2 N}\right]\right| & \leqq n\left|Q_{N} \exp [-i \theta x]-\exp \left[-\rho_{N} \theta^{2 N}\right]\right| \\
& =O\left(n \theta^{2 N+1}\right) .
\end{aligned}
$$

Therefore the diflerence between (33) and (34) is dominated independently of $r$ by 


$$
\begin{aligned}
& \text { constant } \int_{0}^{n^{\lambda}} n \theta^{2 N+1} d \theta+2 \int_{n^{\lambda}}^{\pi} e^{-n A x(1-x) \theta^{2 N}} d \theta+2 \int_{n^{\lambda}}^{\infty} e^{-n \rho N \theta^{2 N}} d \theta \\
& <\text { constant }\left[n^{1+(2 N+2) \lambda}+\exp \left[-A x(1-x) n^{1+2 N \lambda}\right]+\exp \left[-\rho_{N} n^{1+2 N \lambda}\right]\right] .
\end{aligned}
$$

Choosing $\lambda=-1 / 2 N+\varepsilon /(2 N+2)$, the theorem follows.

COROLlary. The constants $D_{0}=D_{0}^{(N)}$ in Theorem 3 all satisfy $D_{0}^{(N)} \geqq 1$.

Proof. Given $N$ and $\varepsilon>0$. Take $n$ large enough so that $\left|c_{0 n}^{(N)}(1 / 2)\right|<\varepsilon$. Define the function $f_{n}(x)$ to equal 1 at $x=1 / 2$, to vanish in the intervals $[1-N, 1 / 2-1 / n]$ and $[1 / 2+1 / n, N]$, and to be linear in the remaining intervals. Its modulus of continuity satisfies $\omega_{0}\left(n^{(1 / 2 N)-1}\right)=1$. Then

$$
\left|B_{n}^{(N)} f_{n}(1 / 2)-f_{n}(1 / 2)\right|=\left|c_{0 n}^{(N)}(1 / 2)-1\right|>(1-\varepsilon) \omega_{0}\left(n^{(1 / 2 N)-1}\right) .
$$

This construction is similar to one devised by Lorentz for the same purpose.

\section{REFERENCES}

1. A. Beurling and H. Helson, Fourier-Stieltjes transforms with bounded powers, Math. Scand. 1 (1953), 20-26.

2. A.Erdélyi, Higher transcendental functions, Bateman Manuscript Project, Vol. 2, McGrawHill, New York, 1953.

3. K. de Leeuw, On the degree of approximation of Bernstein polynomials, Technical Note No. 4, Applied Mathematics and Statistics Laboratory, Stanford Univ., Stanford, Calif., 1959.

4. R. E. Langer, On the asymptotic solutions of ordinary differential equations, with an application to the Bessel functions of large order, Trans. Amer. Math. Soc. 33 (1931), 23-64.

5. G. G. Lorentz, Bernstein polynomials, Univ. of Toronto Press, Toronto, 1953.

6. F. Riesz and B. Sz.-Nagy, Functional analysis, Ungar, New York, 1955.

7. I. J. Schoenberg, Some analytical aspects of the problem of smoothing, Studies and Essays presented to R. Courant, Interscience, New York, 1948.

8. W. G. Strang, Trigonometric polynomials and difference method of maximum accuracy, J. Math. and Phys. 41 (1962), 147-154.

MassachusetTs Institute of TeChNology,

Cambridge, Massachusetts 